Document downloaded from:

http://hdl.handle.net/10251/178656

This paper must be cited as:

Abderrazak, F.; Antonino Daviu, E.; Ferrando Bataller, M. (2020). Power Transfer Efficiency Analyzed using Characteristic Mode Coupling Between Two Parallel Loops. IEEE. 1-5. https://doi.org/10.23919/EuCAP48036.2020.9135229

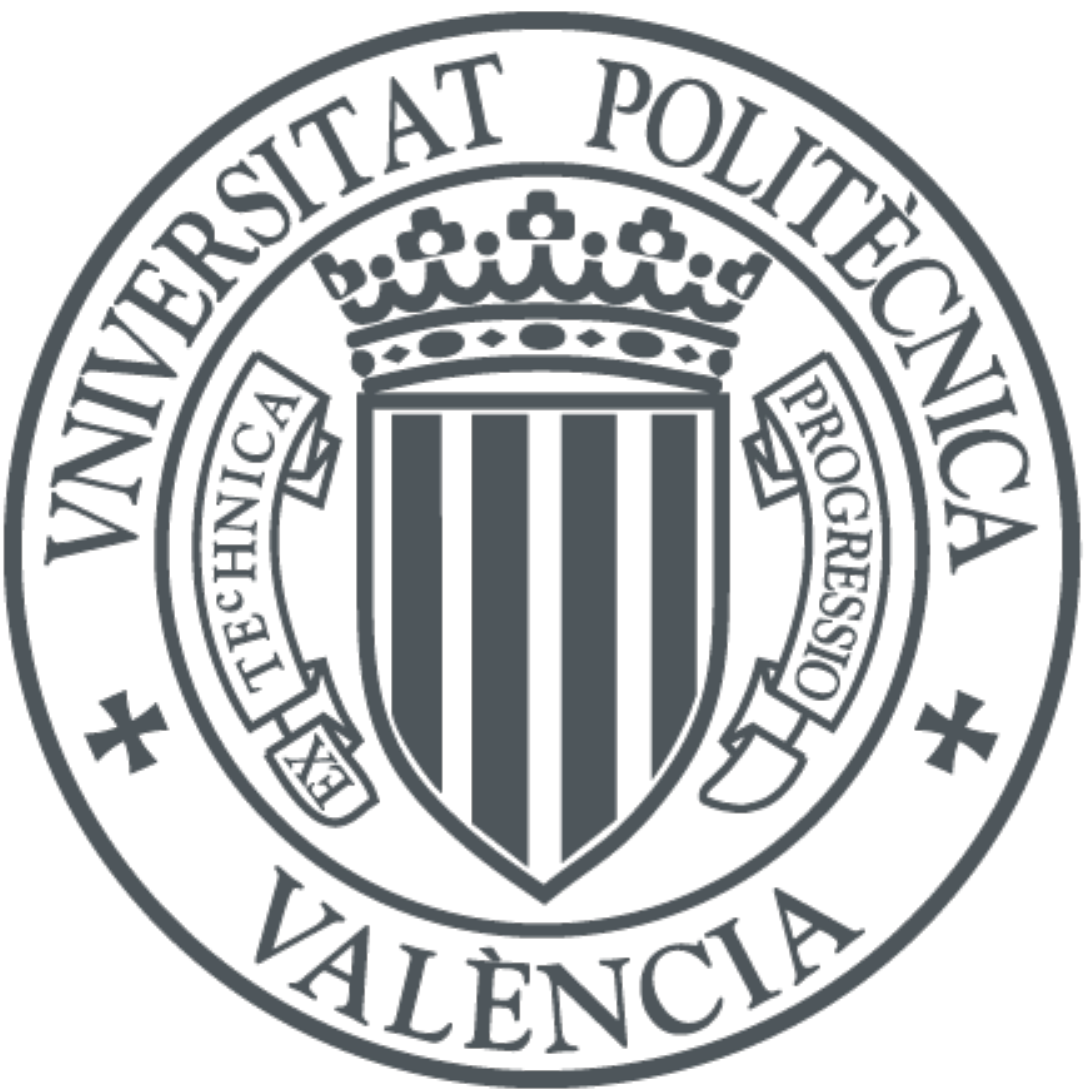

The final publication is available at

https://doi.org/10.23919/EuCAP48036.2020.9135229

Copyright IEEE

Additional Information 


\title{
Power Transfer Efficiency Analyzed using Characteristic Mode Coupling Between Two Parallel Loops
}

\author{
Ferdaous Abderrazak ${ }^{1}$, Eva Antonino-Daviu ${ }^{1}$, Miguel Ferrando-Bataller ${ }^{1}$ \\ ${ }^{1}$ ITEAM, Universitat Politècnica de València, Valencia (46022), Spain \\ ferab@doctor.upv.es, evanda@upvnet.upv.es, mferrand@dcom.upv.es.
}

\begin{abstract}
Independently of any electrical contact, running electronic devices such as smartphones, smart watches, RFID tags etc., is now attainable over small and large distances through Wireless Power Transfer technology. Although, designing systems maintaining appreciable power transfer efficiency still not always achievable. Using two parallel loops, the Theory of Characteristic Modes provides physical insight into the power transfer efficiency. Furthermore, to reach straightforward maximization of the modal power transfer efficiency, the focus of this paper is analyzing the impact of the separation distance and the overlapping between the two antennas on the characteristic modes and their contribution in the total efficiency of the power. The study considers different positions and frequencies of the two parallel antennas.
\end{abstract}

Index Terms - Antenna mode, Characteristic Mode Analysis, Coupling, Coupled Mode Theory, Loop antenna, Odd mode, Power transfer efficiency, Transmission line mode, Wireless power transfer.

\section{INTRODUCTION}

Recent research in wireless communications has shown that the deployment of multiple antennas in the transmitter and the receiver increases drastically the performances of the electronic devices, provided a sufficient separation distance between the main antennas [1], [2], [3]. However, mobile transceivers are limited size devices. Therefore, close spacing between antennas is required. This can profoundly degrade the system performances taking into account the mutual coupling between the different elements [1]. A key feature to analyze the electromagnetic interactions among antennas is to consider them together as one aperture [2]. At close range, the command of resonant frequency has to be accurately determined since the current distribution on one antenna is altered by other antennas [4].

One of the potential technologies based on this concept is Wireless Power Transfer (WPT) that suggests to establish a prospective measure between two radiating elements based on the mutual coupling effect. In instance, charging wirelessly low-power devices and increasing the lifetime of wireless sensor networks are among the widespread applications of this technology [5]. WPT systems transfer power through magnetic induction using mutual induction between two coils [6], since an efficient power transfer up to a distance 8 times their radius has been approved [7].
Various frequencies are approved for WPT applications. Among the existing wireless charging standards, Qi is the leading standard supported by the majority of smartphones manufacturers [8]. For instance, Fig. 1 shows an illustration of a wireless power system which is a special docking station for the ASUS Nexus 7 that starts charging the battery of the tablet or the smartphone through inductance coupling between the two coils. The receiving coil is connected to the mobile device while the transmitting one is connected to the docking station [9]. For Qi chargers, the frequency is located between 110 and $205 \mathrm{KHz}$ for low power up to 5 Watts. For medium power, it is located between 80 and $300 \mathrm{KHz}$.

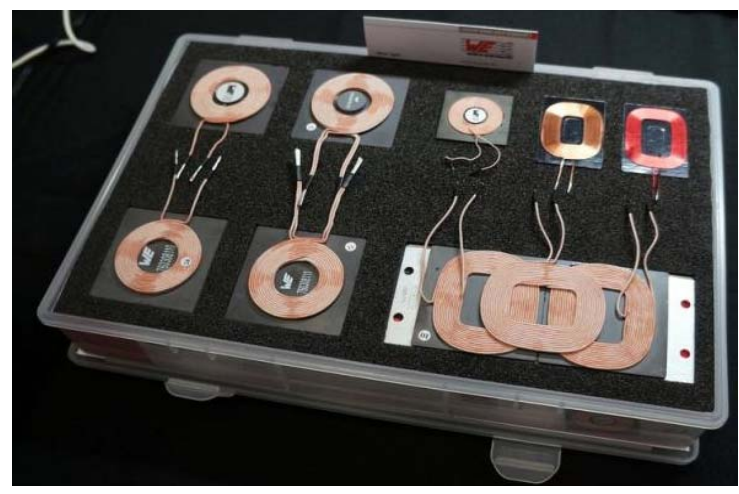

Fig. 1. A special docking station for the ASUS Nexus 7 [9].

For Implantable Medical Devices (IMDs), the most common form of wireless power delivery is low-frequency inductive coupling $(f<10 \mathrm{MHz})$ [10]. More recently, experimental results and modeling have shown that the optimal Power Transfer Efficiency (PTE) into the body is in the UHF band (300 MHz - $3 \mathrm{GHz}$ ) [11], [12]. For example, Industrial Scientific and Medical band, ISM, is clustered around $2.4 \mathrm{GHz}[13]$.

As a matter of fact, several aspects of architectures, hardware designs and implementations of the WPT have been done. Although, they have shown a considerable PTE, their major problem is that they are lacking in physical insight, so real knowledge of the coupling behavior between the two operating antennas is a topic of interest. 
As Characteristic Mode Analysis (CMA) provides an excitation-independent method to analyze electromagnetic properties of arbitrary shaped metallic structures [14], [15], the Theory of Characteristic Modes (TCM) is a favorable tool to investigate the coupling behavior between two loop antennas based on Modal Analysis (MA). Using the impedance matrix $\mathrm{Z}$ of the Method of Moments of a perfectly conducting body, TCM generates Characteristic Modes (CMs) with their associated properties.

This paper proposes a MA of two resonant parallel loop antennas focused on analyzing the coupling and the calculation of the PTE in different frequencies using the software FEKO and MATLAB. Firstly, by changing the separation distance between antennas, an inherent description of the coupling coefficients depending of the $\mathrm{CMs}$ is investigated. Significant difference between the different resonating CMs has been observed by changing the separation between the two identical loops. In the second section, the effect of the separation and overlapping between the two antennas is analyzed. In the last section, a comparison between the different results is reported.

\section{MODAL ANALYSIS OF THE COUPLING AND THE PTE BETWEEN TWO LOOP ANTENNAS}

In this first section, the TCM is introduced based on both mathematical and physical insights. Then, a MA based on the TCM is performed in order to investigate the coupling behavior regarding the separation distance between the two antennas. The coupled mode theory (CMT) is proposed to elucidate the results.

\section{A. Physical Interpretation of Characteristic Modes}

In the TCM, the characteristic modes $J_{n}$, which depend only on the shape and electrical size of the conducting structure, are the real currents on its surface independently of any source. Those currents are calculated consistently using Eq. $1[15]$ :

$$
X\left(J_{n}\right)=\lambda_{n} R\left(J_{n}\right)
$$

where $R$ and $X$ are the real and imaginary parts of the impedance matrix. Although, $X\left(J_{n}\right)$ and $R\left(J_{n}\right)$ are real quantities, typically, the eigen currents $J_{n}$ and their associated eigen values $\lambda_{n}$ are real.

The sign of the eigen values specifies whether the mode is contributing to store magnetic energy $\left(\lambda_{n}<0\right)$ or electric energy $\left(\lambda_{n}>0\right)$. In between, when the eigen value is equal to zero, the exhibited nth mode resonates in a specific frequency denoted as fres, $n$ satisfying:

$$
\lambda_{n}\left(f_{\text {res }, n}\right)=0
$$

Nevertheless, as $\lambda_{n}$ ranges between $-\infty$ to $+\infty$, other presentations of $\lambda_{n}$ are preferred in practice [8], [9], such as the characteristic angle $(M A)$ and the modal significance $(M S)$ :

$$
\begin{gathered}
M A_{n}=180^{\circ}-\arctan \left(\lambda_{n}\right) \\
M S_{n}=\frac{1}{\left|1+j \lambda_{n}\right|}
\end{gathered}
$$

From a physical perspective, $M A_{n}$ presents the phase angle between the characteristic current $J_{n}$ and its characteristic field $E_{n}$. Considering the resonance condition illustrated by Eq. 2, the mode resonates when its modal angle is $180^{\circ}$. The modal significance, given by Eq. 4, represents the normalized amplitude of the current mode. A characteristic mode is resonating when its $M S_{n}$ is equal to 1 . Since, the radiated power is associated to its $M S_{n}$, the radiation bandwidth of the mode can be defined. Indeed, the range of frequencies, when the $M S_{n}$ is above 0.7 , defines the half-power radiating bandwidth.

\section{B. Analysis of the Coupling behavior based on TCM}

A detailed description of the modal resonant frequency is elaborated in this section. In order to observe the effect of coupling on modes in the frequency range from 1 to $4 \mathrm{GHz}$, the separation distance between two identical coplanar loop antennas sharing the same dimensions is varied. The coupled mode theory is applied in order to demonstrate the frequency shift observed in the simulation results.

Before investigating the coupling behavior, let us consider the modal analysis of a single metallic loop antenna. Fig. 2 shows its dimensions, where the inner radius of the loop $(R)$ and the width of the wire $(W)$ are respectively equal to $30 \mathrm{~mm}$ and $1 \mathrm{~mm}$ in the frequency range from 1 to $4 \mathrm{GHz}$.

Fig. 3 shows the characteristic angle $M A_{n}$ associated to the modes over 1 to $4 \mathrm{GHz}$ frequency band. As observed two pairs of degenerated modes sharing identical resonance frequencies can be found. The first pair resonates at 1.65 $\mathrm{GHz}$, while the resonant frequency of the second pair is equal to $3.25 \mathrm{GHz}$.

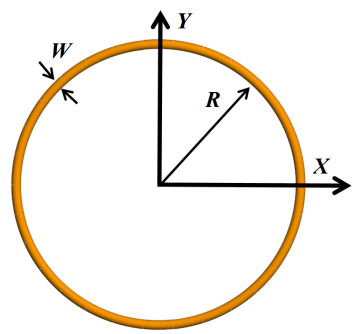

Fig. 2. Geometry of the single loop antenna $(\mathrm{R}=30 \mathrm{~mm}, \mathrm{~W}=1 \mathrm{~mm})$

Additionally, the degenerated modes have the same current distribution with phase difference. As the wire structures provide a clear visualization of the current distribution of modes, Fig. 4 provides schemes of the eigen currents projection along the loop perimeter. $J_{1}$ and $J_{2}$, the first two modes present two current nulls at $\varphi=0^{\circ}$ and at $\varphi=$ 
$90^{\circ}$. The high order modes $J_{4}$ and $J_{5}$ have four current nulls. Based on the symmetry of the current distribution, modes $J_{2}$ and $J_{4}$ represent odd modes while modes $J_{1}$ and $J_{5}$ represent even modes.

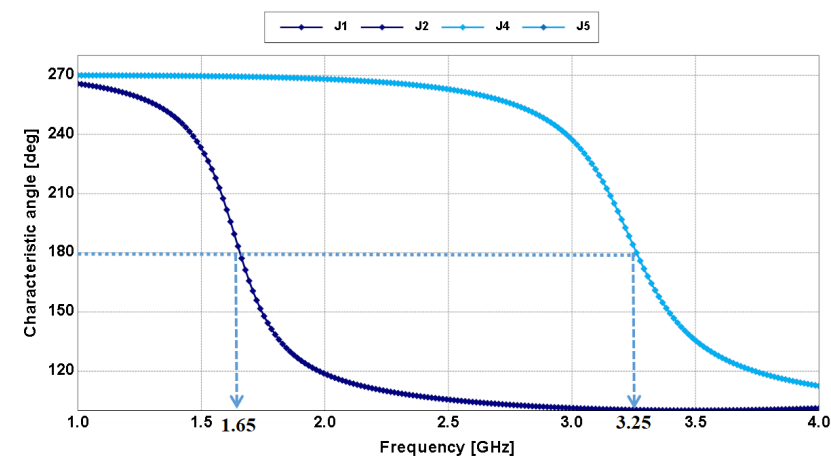

Fig. 3. $M A_{\text {sn }}$ of resonating Modes

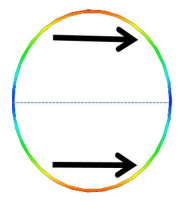

Mode $J_{1}$

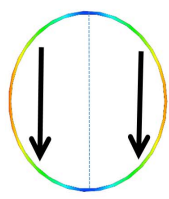

Mode $J_{2}$

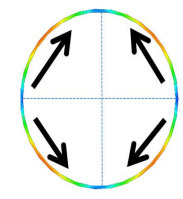

Mode $J_{4}$

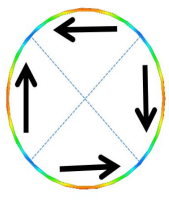

Mode $J_{5}$
Fig. 4. Eigen currents of the wire loop.

Relevant information can be obtained from this analysis while investigating the coupling behavior between two identical loops when varying their separation. For the sake of clarity, the separation increase between the loops has been done gradually, with the antennas placed initially tangent and then sliding to a distance of $2 \lambda$, in order to point out all the variations that resonant modes undergo.

To better illustrate the procedure described above, Fig. 5 shows the results of the computed resonant frequencies for the different CMs when varying the distances between loops. Two regions could be identified. Indeed, the single resonance splits into different resonances when the separation distance reduces and becomes closer to $2 \lambda$. As observed, the antennas are highly coupled when their evanescent fields are overlapped and the coupling can be explained using the coupled mode theory [16]. Eq. 5 presents resonant radian frequencies of the coupled modes basing on CMT:

$$
\omega=\frac{\omega_{1}+\omega_{2}}{2} \pm \sqrt{\left(\frac{\omega_{1}+\omega_{2}}{2}\right)^{2}+k^{2}}
$$

where $\omega_{1}$ and $\omega_{2}$ are the radian resonant frequencies of the odd and even modes having the same current distribution and $k$ is the coupling coefficient. At a large distance, the coupling effect vanishes and the coupling coefficient is equal to zero. This is consistent with simulations results as the two set of CMs converge to resonant frequencies of the single loop antenna, which are $1.65 \mathrm{GHz}$ and $3.25 \mathrm{GHz}$.

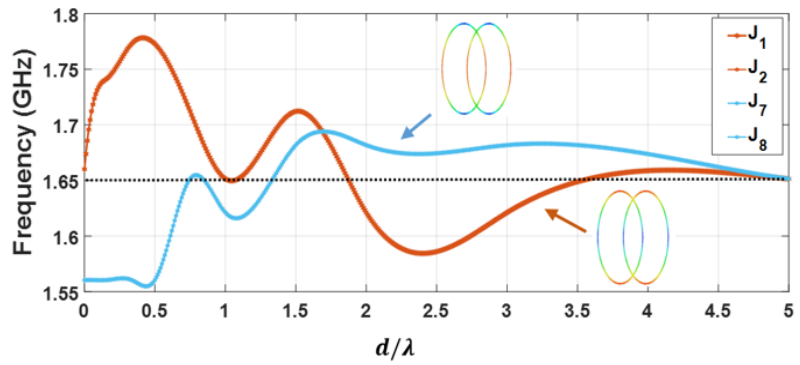

(a)

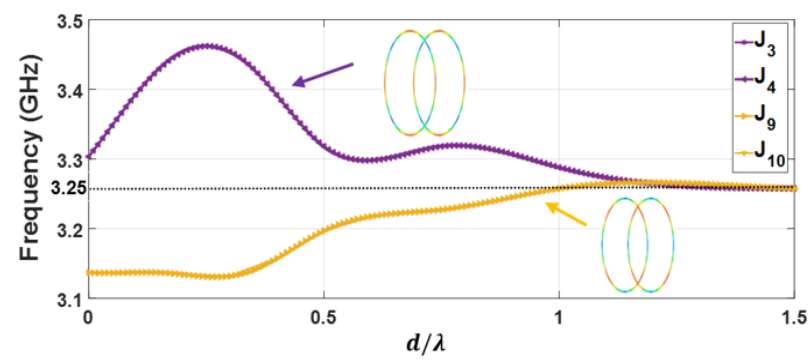

(b)

Fig. 5. Frequency shift of the two group of CMs: (a) First set of CMs, (b) Second Set of CMs.

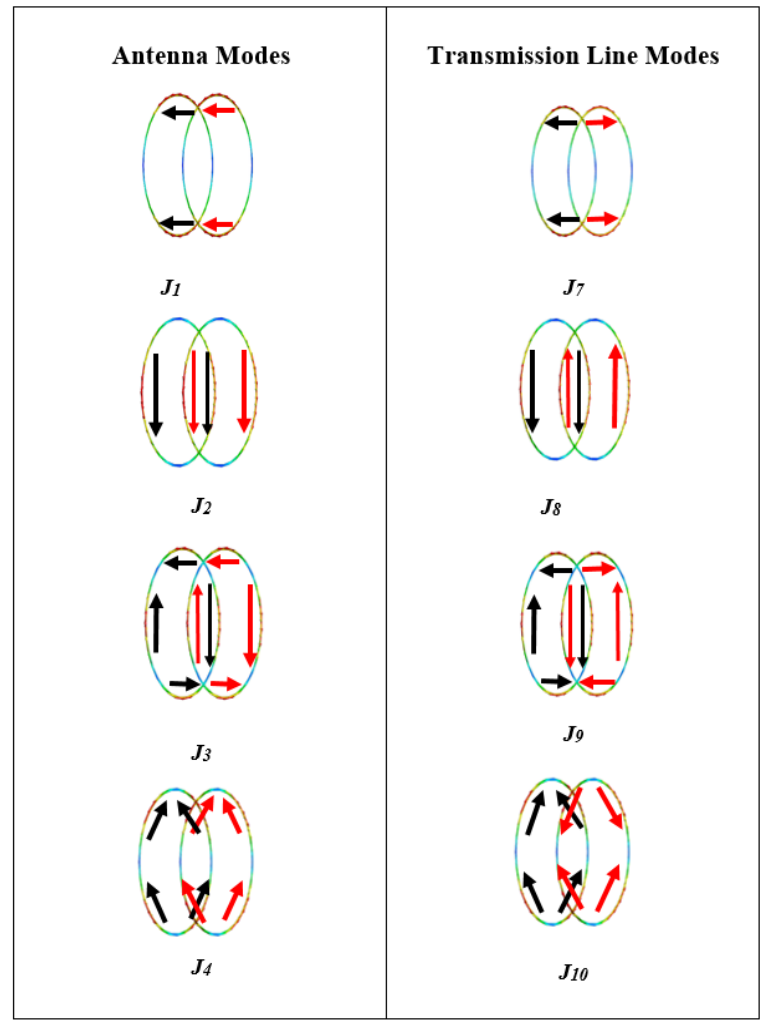

Fig. 6. Current distributions of the two coupled loop antennas at $1.65 \mathrm{GHz}$ for $J_{1}, J_{2}, J_{7}$ and $J_{8}$, and at $3.25 \mathrm{GHz}$ for $J_{3}, J_{4}, J_{9}$ and $J_{10}$.

Based on different approaches, the existence of these antenna modes and transmission line modes is very 
important [15]. As seen in Fig. 6, when the two loops are coupled in transmission-line way $\left(J_{7}, J_{8}, J_{9}\right.$ and $\left.J_{10}\right)$, the radiation will decrease due to the current cancellation. Therefore, antenna modes should be excited since they provide broader bandwidth and better radiation. In this section the coupling effect on the different resonant frequencies of different CMs has been analyzed. In the third section, the PTE will be investigated in terms of the separation and the overlapping distances.

\section{CMA of PTE of the two parallel loops}

A detailed description of the total and the modal power transfer efficiencies are given in this section for the two parallel loops in the frequency range from 350 to $600 \mathrm{MHz}$. In order to observe the effect of distance on the PTE, the separation distance was varied gradually. For the overlapping, the PTE was analyzed in two specific separation distances while changing the position of the upper loop. Before investigating the power transfer efficiency, let us consider the CMA of the two parallel loop antennas at a specific separation distance. Fig. 7 shows their dimensions, where the inner radius of the loop $(R)$ and the width of the wire $(W)$ are respectively equal to $115 \mathrm{~mm}$ and $1 \mathrm{~mm}$ and the separation distance is $S$.

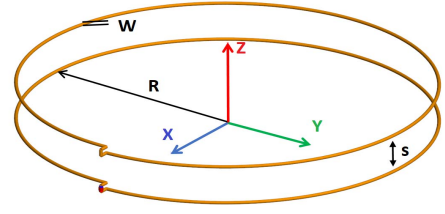

Fig. 7. The dimensions of the two parallel loops.

For instance, for a specific separation distances $S_{l}$ equal to $20 \mathrm{~mm}$, Fig. 8 shows the $\mathrm{MA}_{\mathrm{n}}$ associated to the modes over 350 to $600 \mathrm{MHz}$ frequency band. Drawing an analogy with the last section, two pairs of degenerated modes sharing identical resonant frequencies can be found. The first pair resonates at $416 \mathrm{MHz}$, while the resonant frequency of the second resonant $\mathrm{CMs}$ is equal to $459 \mathrm{MHz}$. Fig. 9 illustrates a clear visualization of the current distribution of the observed CMs in their resonances.

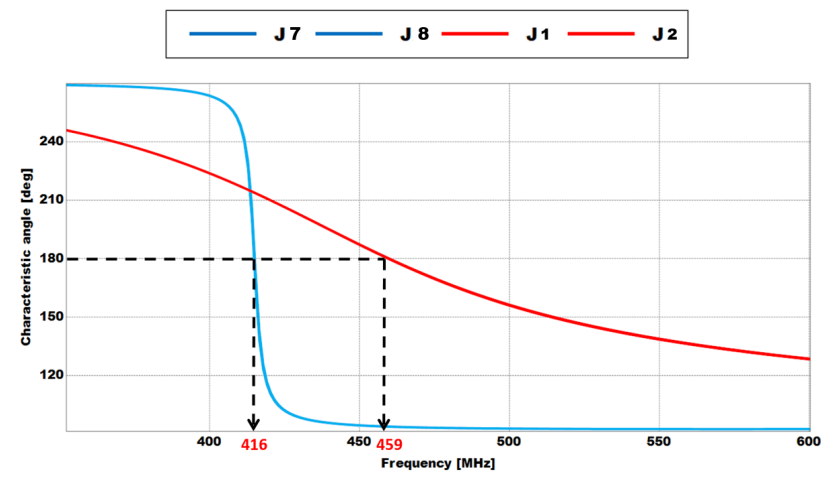

Fig. 8. MA of the two parallel loops at a separation distance $\mathrm{S}=50 \mathrm{~mm}$.
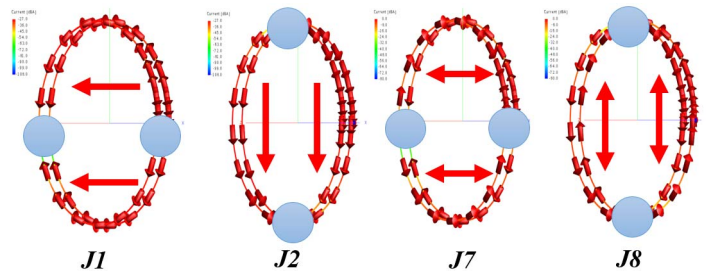

Fig. 9. Current distributions of the resonating $\mathrm{CMs}$

Relevant information can be obtained from this analysis while investigating the contribution of each resonating CM in the total PTE between the two parallel loops. The PTE between two coupled antennas $\eta$ is derived in terms of the dissipated power at the load of the receiving antenna divided by the accepted power into the transmitting antenna [16]:

$$
\eta=\frac{P_{R_{L}}}{P_{\text {in }}}=\frac{\left|I_{2}\right|^{2} R_{L}}{\left|I_{1}\right|^{2} R_{\text {in }}}
$$

Where $P_{R L}$ is the power dissipated in the load $R_{L}$ of the receiving antenna, $P_{i n}$ is the accepted power in the transmitting antenna, $R_{i n}$ is the input resistance, and $I_{l}$ and $I_{2}$ denote respectively the currents in the transmitter and the receiver.

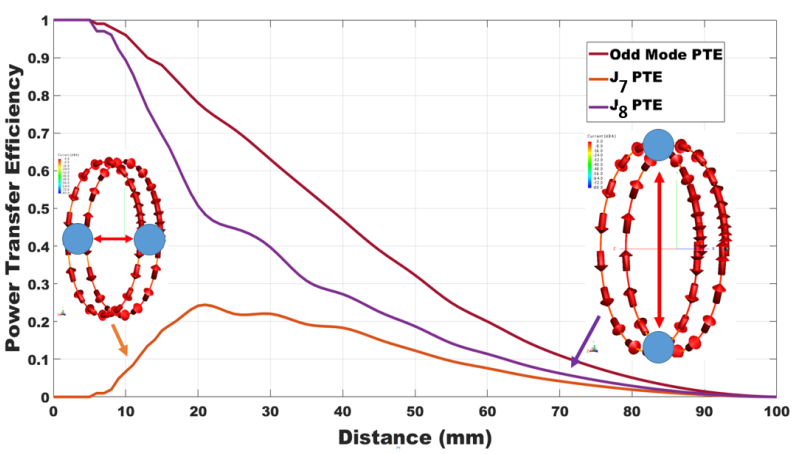

Fig. 10.PTEs of the odd mode, $J_{7}$ and $J_{8}$.

Using the software FEKO, the accepted power in the transmitting and receiving antennas could be pinpointed. By requesting the CMA, the contribution of each $\mathrm{CM}$ in both powers will be deduced. Yet, the modal PTE is calculated. Fig. 10 shows the PTE of the coupled antennas as well as the modal PTEs of the efficient resonating CMs. During the parametric study, the contribution of antenna $J_{1}$ and $J_{2}$ was very limited and did not exceed the $3 \%$. The simulation result shows three distinct spatial regions. The first one holds for up to $20 \mathrm{~mm}$ where the contribution of $J_{8}$ is more important than $J_{7}$. This is reasonable since the odd mode is a transmission line mode when drawing an analogy with the TCM. In the second region, a power decay can be observed and $J_{8}$ present a slightly higher contribution compared to $J_{7}$. The coupling decreases and the losses in the antennas deteriorate the PTE in the third region.

Those results would be more significant if we compare them with other positions of the two wire loops. In particular, 
we will see the effect of overlap on the contribution of CMs and on the PTE in two different separation distances. The presentation of the PTE is limited on $J_{8}$ since it is the prevailing mode and the contribution of other modes including $J_{7}$ does not transcend $2 \%$. Indeed, a minimal increase in the overlap distance cancels the contribution of $J_{7}$ and foster the contribution of $J_{8}$. Concerning the effect of overlapping on PTE, it shares the same effect as the separation as we can distinguish the three different regions. Although, the PTE is lesser affected by the overlapping compared to the separation. For instance, at a separation distance equal to $80 \mathrm{~mm}$, the PTE is equal to 0.05 . On the other hand, for the same distance of overlap, the PTE is equal to 0.24 .

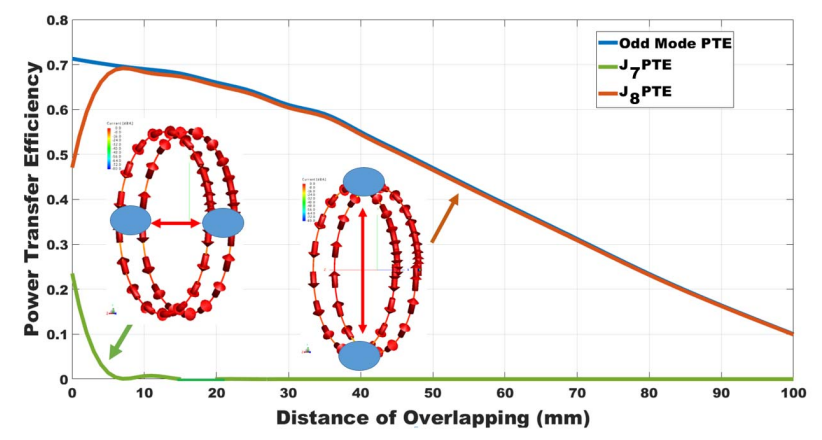

Fig. 11. PTE of the odd mode and $J_{8}$ upon the overlap at separation distance equal to $25 \mathrm{~mm}$.

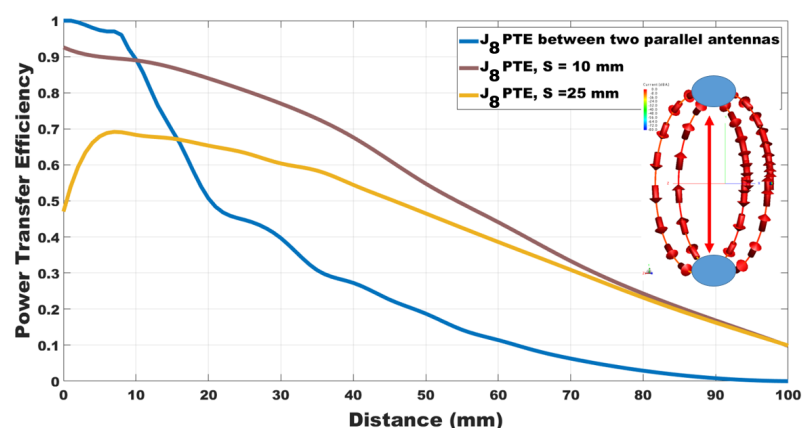

Fig. 12.PTE of $J_{8}$ in three proposed cases.

\section{CONCLUSION}

It has been shown that the Theory of Characteristic Modes is able to provide a physical investigation on the coupling and on the Wireless Transfer Efficiency. As a general rule, the coupling doesn't affect CMs the same way. Translated to TCM, the odd mode is a transmission line mode with nulls at $\varphi=0^{\circ}$. This $\mathrm{CM}$ presents usually the higher contribution. Besides, the efficiency of the power transfer is more susceptible to the increase of the separation distance then the raise of the overlap. This study is suitable for wire loop antennas dedicated especially for WPT. For example, wireless chargers are usually composed of more than one coil. The distance between the coils as well as the distance of separation between the transmitting and receiving antennas must be taken into consideration. These parameters are of great importance to ensure an efficient PTE. Further studies on angular misalignment for different characteristic modes will be presented in the conference.

\section{ACKNOWLEDGMENT}

This work has been supported by the Spanish Ministry of Science, Innovation and Universities (Ministerio Ciencia, Innovación y Universidades) under the project TEC201678028-C3-3-P.

\section{REFERENCES}

[1] L. Kundu, "Information Theoretic Limits on MIMO Antennas," Ph.D. Thesis, 2016

[2] A. Jabire, H. X. Zheng, A. Abdu and Z. Song, "Characteristic mode analysis and design of wide band MIMO antenna consisting of metamaterial unit cell," in Electronics, vol. 8, no 1, pp. 68, January 2019

[3] Y. Wu, K. Ding, B. Zhang, J. Li, D. Wu, and K. Wang, "Design of a compact UWB MIMO antenna without decoupling structure," in Antenna Prop. 2018, 2018

[4] K. W. Qian, G. L. Huang, J. J. Liang, B. Qian and T. Yuan, “An LTCC interference cancellation device for closely spaced antennas decoupling," in IEEE Access, vol. 6, p. 68255-68262, November 2018

[5] Z. Ding, C. Zhong, D. W. K. Ng, M. Peng, H. A. Suraweera, R. Schober and H. V. Poor, "Application of smart antenna technologies in simultaneous wireless information and power transfer," in IEEE Communications Magazine, vol. 53, no. 4, pp. 86-93, April 2015

[6] P. S. Riehl, "Dual-mode wireless power receiver," in U.S. Patent, January 2018

[7] A. Kurs, A. Karalis, R. Moffatt, J. D. Joannopoulos, P. Fisher and M. Soljac, "Wireless power transfer via strongly coupled magnetic resonances," in Science Magazine, vol. 317, no 5834, p. 83-86, July 2007

[8] X. Lu, P. Wang, D. Niyato, D. I. Kim and Z. Han, "Wireless charging technologies: Fundamentals, standards, and network applications," in IEEE Communications Surveys \& Tutorials, vol. 18, no 2, p. 14131452, November 2015

[9] "Wireless charging and docking station for the ASUS Nexus 7," in Bashny.net, 2012. [Online].Available: https://bashny.net/t/en/272903. [Accessed: 18-Apr-2019]

[10] D. K. Freeman, and S. J. Byrnes, "Optimal Frequency for Wireless Power Transmission Into the Body: Efficiency Versus Received Power," in IEEE Transactions on Antennas and Propagation, vol. 67, no 6, pp. 4073-4083, June 2019

[11] A. S. Y. Poon, S. O'Driscoll, and T. H. Meng, "Optimal frequency for wireless power transmission into dispersive tissue," IEEE Trans. Antennas Propag., vol. 58, no. 5, pp. 1739-1750, May 2010

[12] D. Nikolayev, M. Zhadobov, M. Karban, and R. Sauleau, "Electromagnetic radiation efficiency of body-implanted devices," Phys. Rev. Appl., vol. 9, no. 2, 2018, Art. no. 024033

[13] C. F. Chiasserini, and R. R. Rao, "Coexistence mechanisms for interference mitigation in the $2.4-\mathrm{GHz}$ ISM band," in IEEE Transactions on Wireless Communications, vol. 2, no 5, pp. 964-975, September 2003

[14] M. Capek, V. Losenicky, L. Jelinek and M. Gustafsson, "Validating the characteristic modes solvers," in IEEE Trans. Antennas Propag., vol. 65, no 8, p. 4134-4145, May 2017.

[15] E. Antonino-Daviu, "Analysis and design of antennas for wireless communications using modal methods," Doctoral dissertation, February 2008

[16] Y. Kim, and H. Ling, "Investigation of coupled mode behaviour of electrically small meander antennas," in Electronics letters, vol. 43, no 23, pp. 1250-1252, November 2007 